\title{
Molecular clustering based on ER $\alpha$ and EIG121 predicts survival in high-grade serous carcinoma of the ovary/peritoneum
}

\author{
Matthew P Schlumbrecht ${ }^{1}$, Su-Su Xie ${ }^{2}$, Gregory L Shipley ${ }^{3}$, Diana L Urbauer ${ }^{4}$ and \\ Russell R Broaddus ${ }^{2}$
}

${ }^{1}$ Department of Gynecologic Oncology, The University of Texas M.D. Anderson Cancer Center, Houston, TX, USA; ${ }^{2}$ Department of Pathology, The University of Texas M.D. Anderson Cancer Center, Houston, TX, USA; ${ }^{3}$ Department of Integrative Biology and Pharmacology, The University of Texas Health Science Center at Houston, Houston, TX, USA and ${ }^{4}$ Division of Quantitative Sciences, The University of Texas M.D.

Anderson Cancer Center, Houston, TX, USA

\begin{abstract}
Assessment of estrogen receptor (ER) expression by immunohistochemistry has yielded inconsistent results as a prognostic indicator in ovarian carcinoma. In breast and endometrial carcinomas, panels of estrogeninduced genes have shown improved prognostic capability over the use of ER immunohistochemistry alone. For both breast and endometrial cancers, overexpression of estrogen-induced genes is associated with better prognosis. We hypothesized that analysis of a panel of estrogen-induced genes can predict the outcome in ovarian carcinoma and potentially differentiate between tumors of varying hormonal responsiveness. From a cohort of 219 women undergoing ovarian cancer surgery from 2004 to 2007, 83 patients were selected for inclusion. All patients had advanced stage ovarian/primary peritoneal high-grade serous carcinoma and underwent primary surgical debulking, followed by adjuvant treatment with platinum and taxane agents. The expression of $E R \alpha$ and six genes known to be induced by estrogen in the female reproductive tract (namely EIG121, sFRP1, sFRP4, RALDH2, PR, and IGF-1) was measured using quantitative RT-PCR. Unsupervised cluster analyses were used to categorize patients as high or low gene expressors. Gene expression results were then compared with those for ER immunohistochemistry. Clusters were compared using $\chi^{2}$ analyses, and Cox proportional hazards models were used to evaluate survival outcomes. The median follow-up time was 38.7 months (range: 1-68). A cluster defined by ElG121 and ER $\alpha$ segregated tumors into distinct groups of high and low gene expressors. Shorter overall survival (OS) was associated with high gene expression (HR 2.84 (1.11-7.30), $P=0.03)$, even after adjustment for other covariates. No difference in ER immunohistochemistry expression was noted between gene clusters. In contrast to other hormonally driven cancers, high expression of $E R \alpha$ and the estrogen-induced gene EIG121 predicts shorter OS in patients with high-grade serous ovarian carcinoma. Such a biomarker panel may potentially be used to guide management with estrogen antagonists in this patient population.
\end{abstract}

Modern Pathology (2011) 24, 453-462; doi:10.1038/modpathol.2010.211; published online 19 November 2010

Keywords: estrogen-induced genes; hormone antagonism; ovarian high-grade serous carcinoma

Ovarian cancer is the fifth most common cause of cancer death in American women. ${ }^{1}$ Carcinoma of the ovary is typically diagnosed in women older than

Correspondence: Dr RR Broaddus, MD, PhD, Department of Pathology, The University of Texas M.D. Anderson Cancer Center, 1515 Holcombe Boulevard, Unit 85, Houston, TX 77030, USA.

E-mail: rbroaddus@mdanderson.org

Received 8 June 2010; revised 25 August 2010; accepted 30 August 2010; published online 19 November 2010
50 years of age, and the majority of cancers are advanced at the time of diagnosis. ${ }^{1}$ The majority of ovarian cancer types are epithelial in origin, and of these, high-grade serous carcinoma is the most common. For the majority of patients, the treatment of high-grade serous carcinoma is surgical debulking, followed by adjuvant chemotherapy with combination platinum and taxane agents. The 5-year overall survival (OS) for women with highgrade serous carcinoma is $<30 \%$, due in part to its 
asymptomatic progression from localized tumor to metastatic disease, high rate of recurrence, development of chemoresistance, and the paucity of agents effective at treating recurrent disease..$^{1-3}$

As ovarian function is, at least in part, hormonally regulated, it is generally presumed that ovarian carcinoma should be somewhat responsive to hormonal manipulation. Given the success of hormone-manipulating agents in the treatment of breast, endometrial, and prostate carcinomas, incorporating agents aimed at antagonizing hormonedependent proliferative pathways into the ovarian cancer treatment algorithm has been proposed. Agents targeting estrogen pathways are of particular interest, especially as the inhibition of estrogen in estrogen receptor (ER)-positive breast cancers translates into reduced risk of recurrence and increased survival. ${ }^{4}$ Despite the fact that $>60 \%$ of ovarian cancers demonstrate ER positivity, ${ }^{5}$ inhibition of estrogen-associated pathways has not translated into significant improvements in patient outcomes. In a meta-analysis of 18 ovarian cancer trials that treated patients with tamoxifen, a selective ER modulator, the collective response rate was only $13 \%{ }^{6}$ Pretreatment determination of tumor ER status has also demonstrated poor predictive ability of response to treatment. For example, Schwartz et al ${ }^{7}$ performed a prospective trial of chemotherapy with or without tamoxifen in patients with advanced stage ovarian cancer and noted that there was no relation between hormone receptor status and survival outcomes. More recent studies evaluating the activity of aromatase inhibitors in ER-positive patients have reported response rates between 3 and $17 \%$, with stable disease achieved in up to $26 \%{ }^{8,9}$ Taken together, these investigations suggest that there is a subset of women with ovarian cancer who will have some degree of response to hormone antagonism, but ER immunohistochemistry may not be a sufficient means of identifying these patients.

An alternative to ER immunohistochemical assessment is to evaluate genes known to be induced by estrogen, a strategy that has resulted in improved capability to segregate tumors based on hormone sensitivity in other malignancies. In breast cancer, quantitative examination of estrogen-regulated genes helps to detect subgroups within ER-positive tumors with differing survival parameters, even when accounting for tumor characteristics, such as lymph-node positivity, tumor size, and the use of chemotherapy. ${ }^{10}$ Using specimens provided by several different investigators, a gene panel proposed by $\mathrm{Oh}$ et $a l^{10}$ accurately predicted patients with invasive breast ductal carcinoma who had markedly different relapse-free survival. Similar findings have been reported in endometrial cancer. In 2009, Westin et $a l^{11}$ described a panel of estrogeninduced genes in patients with endometrial carcinoma, which identified two distinct clusters based on the degree of gene expression. Higher estrogen-regulated gene expression was predictive of improved recurrence-free survival (RFS) and was able to distinguish between high/intermediateand low-risk tumors with a false-negative rate of only $4.8 \% .^{11}$

Given the findings in breast and endometrial carcinomas that estrogen-regulated genes demonstrate prognostic capability, it is possible that analyzing estrogen-regulated gene expression may have similar utility for patients with ovarian cancer. Determining which subset of women with ovarian cancer who may potentially respond to estrogen antagonism would afford the oncologist the ability to initiate such treatment earlier in the disease course, either alone or in combination with other therapies. Our primary aim was to quantify the expression of estrogen-induced genes in a cohort of women with the most common ovarian cancer, highgrade serous carcinoma, and to determine whether differential expression was predictive of clinical outcomes. Secondarily, we compared gene expression with immunohistochemical assessment of ER, the current standard for judging hormone sensitivity, to determine whether immunohistochemistry accurately predicts tumor molecular profiles. We hypothesized that this examination of estrogeninduced genes would identify subsets of patients with different clinical characteristics and distinct survival outcomes. As higher estrogen-induced gene expression portends improved prognosis in other hormone-sensitive tumors, we expected that a similar relationship would be observed in this cohort of ovarian cancer patients.

\section{Materials and methods}

\section{Patient Selection and Clinical Data Acquisition}

After obtaining IRB approval, a review of the institutional tumor bank identified 219 patients from whom ovarian or primary peritoneal carcinoma specimens were obtained at the time of tumorreductive surgeries between 2004 and 2007. Pathological diagnoses were made by gynecological pathologists after microscopic review of hematoxylin and eosin-stained slides derived from surgical specimens containing ovarian or primary peritoneal carcinomas. Patient clinical characteristics were obtained by a review of electronic medical records and included date of birth, race, anthropometric variables, date of surgical staging, debulking status, primary and secondary chemotherapy regimens, date of recurrence, date of last follow-up, and disease status at last follow-up. Both clinical and pathological features were used to determine inclusion criteria. Patients selected for inclusion in the study demonstrated only advanced stage (III or IV), high-grade serous ovarian or primary peritoneal carcinoma. In addition, all patients received treatment with platinum and taxane agents as firstline adjuvant chemotherapy. Specific exclusion criteria included treatment with neoadjuvant 
chemotherapy, consolidation/maintenance chemotherapy, and first-line treatment regimens that were experimental protocols or not platinum based. Body mass indices (BMIs) were categorized by World Health Organization definitions of normal weight, overweight, and obese. Chemotherapy resistance was determined using Gynecologic Oncology Group criteria, which include the following: (1) disease progression while on a first-line platinumbased regimen; (2) tumor progression within 6 months of completion of platinum-based therapy; and (3) persistent clinically measurable disease with best response as stable disease at the completion of first-line therapy. ${ }^{12}$

\section{Gene Selection and RNA Preparation}

Seven genes (namely $E R \alpha, P R, E I G 121, I G F-1$, $R A L D H 2$, sFRP1, and $s F R P 4)$ were included for transcript quantification by quantitative RT-PCR (qRT-PCR). Our research group has previously used a genomics approach to identify that EIG121, $R A L D H 2, s F R P 1$, and $s F R P 4$ are highly induced by estrogen in the human female reproductive tract. ${ }^{11,13,14} P R$ and $I G F-1$ are classical estrogeninduced genes. ${ }^{15,16}$ Transcript analysis of ER $\alpha$ was included for subsequent comparisons with ER immunohistochemistry studies. After microscopic confirmation of tumor histology and presence of $>70 \%$ viable tumor, RNA was extracted from frozen tissues, prepared using the TriReagent (Molecular Research Center, Cincinnati, OH, USA), and precipitated with isopropyl alcohol. Ultraviolet spectrometry was used to confirm adequate concentrations of RNA in each prepared specimen. RNA was applied to RNeasy spin columns (Qiagen, Valencia, CA, USA), eluted, and treated with $10 \%$ RNAse-free DNAse solution. Specimens were incubated for $30 \mathrm{~min}$ at $37^{\circ} \mathrm{C}$ and subsequently treated for $10 \mathrm{~min}$ at $75^{\circ} \mathrm{C}$ to inactivate DNAse I. Postincubation RNA was stored at $-80^{\circ} \mathrm{C}$ until qRT-PCR was performed.

\section{Reverse Transcription and Real-Time PCR}

Probes and primers (TaqMan probe) for the gene panel are shown in Table 1. Aliquots (100 ng) of each RNA were reverse transcribed in quadruplicate (including a no reverse transcriptase control) with a $300 \mathrm{nM}$ assay-specific reverse primer, $4 \mathrm{mM} \mathrm{MgCl}_{2}$, $500 \mu \mathrm{M}$ dNTPs, and 10 Units of MMLV Superscript II reverse transcriptase (Invitrogen, Carlsbad, CA, USA) at $50^{\circ} \mathrm{C}$ for $30 \mathrm{~min}$, followed by $72^{\circ} \mathrm{C}$ for $5 \mathrm{~min}$. In all, $40 \mu \mathrm{l}$ of PCR mix containing $1 \times$ PCR buffer, $300 \mathrm{nM}$ specific forward and reverse primers, $4 \mathrm{mM} \mathrm{MgCl}_{2}$, Taq DNA polymerase, and $100 \mathrm{nM}$ fluorogenic probe was then added to each $10-\mu \mathrm{l}$ RT reaction. Amplification was performed using the ABI Prism 7700 Sequence Detection System (Applied Biosystems, Foster City, CA, USA) at $95^{\circ} \mathrm{C}$ for $1 \mathrm{~min}$, followed by 40 cycles of a 12 -s step at $95^{\circ} \mathrm{C}$ and a 1-min step at $60^{\circ} \mathrm{C}$. Synthetic RNA or singlestrand DNA amplicon standards were serially diluted in water containing $100 \mathrm{ng} / \mu \mathrm{l}$ yeast tRNA (Invitrogen).

\section{Immunohistochemistry}

Immunohistochemistry for ER $\alpha$ (clone 6F11, 1:35; Novacastra/Leica Microsystems, Chicago, IL, USA) was performed using standard histological techniques. Antigen retrieval was accomplished with citrate buffer for $30 \mathrm{~min}$ at $100^{\circ} \mathrm{C}$. Stained slides were microscopically reviewed in a blinded manner by the study primary author (MPS) and a gynecological pathologist (RRB). ER $\alpha$ protein expression was scored semi-quantitatively as percent tumor with $2+$ or $3+$ nuclear staining. Tumors were considered positive if $\geq 10 \%$ of tumor cell nuclei demonstrated strong $(2+$ or $3+)$ expression.

\section{Statistical Analyses}

Statistical analyses were performed using STATA 10.0 (StataCorp, College Station, TX, USA) and SPSS 17.0 (SPSS, Chicago, IL, USA). Summary statistics were generated to describe the study population. The Wilcoxon rank-sum test was used to compare mean gene expression in relation to clinical variables, and linear regression was used to compare gene expression with continuous clinical variables (BMI, age). $\chi^{2}$ analyses were used to describe the relationship between categorical variables, and logistic regression was used to determine associations between clinical variables and categorical outcomes. RFS and OS for each gene were analyzed using Cox proportional hazards models and the Kaplan-Meier method. RFS was defined as the interval between initial surgical treatment and date of recurrence. OS was defined as the interval between initial surgical treatment and date of last follow-up or death. A $P$-value of $<0.05$ was considered statistically significant, and all tests were two sided.

Unsupervised cluster analyses were performed for each gene separately, and in various permutations, to include all six estrogen-induced genes and $E R \alpha$. Specific clusters were selected based on their potential ability to segregate tumors into distinct groups, and analyzed to compare differences in survival, outcome, and demographic characteristics between these groups. The characteristics between cluster groups were compared using $\chi^{2}$ or Fisher's exact analyses, when appropriate. Univariate Cox proportional hazards models were used to analyze gene associations between survival, gene expression clusters, and other patient clinical features. Variables with $P$-values $<0.15$ in the univariate analysis were included in the multivariate model. 
Table 1 Probes and primers for real-time PCR

\begin{tabular}{|c|c|c|}
\hline Transcript & Taqman primers and probe & Accession number \\
\hline hEIG121 & $\begin{array}{l}\text { 3210(+)CTTGCATAGCACCTTTGCAAG } \\
\text { 3135(-)CAGTGGGTGTTGCAGGATG } \\
\text { 3232(+)FAM-CYGCGGCGATTTGGGTGCC-BHQ1 } \\
\text { Lowest quantifiable level }=240 \text { molecules } \\
\text { Average assay efficiency }=90 \%\end{array}$ & NM_020777 \\
\hline$h I G F-1$ & $\begin{array}{l}\text { 146(+)GCAATGGGAAAAATCAGCAG } \\
\text { 237(-)GAGGAGGACATGGTGTGCA } \\
\text { 217(-)FAM-CTTCACCTTCAAGAAATCACAAAAGCAGCA-BHQ1 } \\
\text { Lowest quantifiable level }=160 \text { molecules } \\
\text { Average assay efficiency }=99 \%\end{array}$ & M26544 \\
\hline$h R A L D H 2$ & $\begin{array}{l}\text { 2002(+)AGGCCCTCCTCGCTCAC } \\
\text { 2071(-)TCTGCCCCAGAATGAGCTC } \\
\text { 2021(+)FAM-ACCCCTCCCTCTCTTCCAAGGAGATC-BHQ1 } \\
\text { Lowest quantifiable level }=210 \text { molecules } \\
\text { Average assay efficiency }=96 \%\end{array}$ & NM_003888 \\
\hline hsFRP1 & $\begin{array}{l}\text { 720(+)GAGCCGGTCATGCAGTTCT } \\
\text { 786(-)CCTCCGGGAACTTGTCACA } \\
\text { 740(+)FAM-CGGCTTCTACTGGCCCGAGATCG-BHQ1 } \\
\text { Lowest quantifiable level }=210 \text { molecules } \\
\text { Average assay efficiency }=95 \%\end{array}$ & NM_003012 \\
\hline$h s F R P 4$ & $\begin{array}{l}\text { 1175(+)GCGCACCAGTCGTAGTAATCC } \\
\text { 1246(-)TTCTTGGGACTGGCTGGTT } \\
\text { 1202(+)FAM-ACCAAAGGGAAAGCCTCCTGCTCC-BHQ1 } \\
\text { Lowest quantifiable level }=200 \text { molecules } \\
\text { Average assay efficiency }=99 \%\end{array}$ & AF026692 \\
\hline$h E R \alpha$ & $\begin{array}{l}\text { 1394(+)TACTGACCAACCTGGCAGACAG } \\
\text { 1490(-)TGGACCTGATCATGGAGGGT } \\
\text { 1466(-)FAM-TCCACAAAGCCTGGCACCCTCTTC-BHQ1 } \\
\text { Lowest quantifiable level }=150 \text { molecules } \\
\text { Average assay efficiency }=95 \%\end{array}$ & NM_000125 \\
\hline$h P R$ & $\begin{array}{l}\text { 2689(+)GAGCACTGGATGCTGTTGCT } \\
\text { 2754(-)GGCTTAGGGCTTGGCTTTC } \\
\text { 2710(+)FAM-TCCCACAGCCAGTTGGGCGTTC-BQH1 } \\
\text { Lowest quantifiable level }=220 \text { molecules } \\
\text { Average assay efficiency }=93 \%\end{array}$ & NM_000926 \\
\hline h18s & $\begin{array}{l}\text { Primers: } \\
(1335+) \text { CGGCTTAATTTGACTCAACAC } \\
\text { (1401-)ATCAATCTGTCAATCCTGTCC } \\
\text { Probe: } \\
\text { (1359+)AAACCTCACCCGGCCCG } \\
\text { Amplicon-68 bases in length }\end{array}$ & M10098 \\
\hline
\end{tabular}

The Kaplan-Meier method and log-rank test were used to compare survival outcomes.

The Wilcoxon rank-sum test was used to compare the percentage of ER immunohistochemical expression between tumor samples obtained from different clusters. Fisher's exact test was used to compare clusters when protein expression was characterized categorically. All tests were two sided, and a $P$-value of $<0.05$ was considered statistically significant.

\section{Results}

A total of 219 women underwent surgery for ovarian or primary peritoneal carcinoma during the study interval. Application of the inclusion criteria resulted in a relatively homogenous cohort for study numbering 113 patients of which frozen tissues for
83 were available from the institutional Gynecologic Oncology Tumor Bank. Patients were followed for a median of 38.7 months (range: 0.5-67.8) from the time of their cancer diagnoses. The demographic features of the cohort of 83 patients are summarized in Table 2. The mean age at diagnosis was 62.6 years (range: $34.5-85.9$ ), and the majority of patients were Caucasian. A significant proportion of patients were overweight or obese $(42.2 \%)$, and $>18 \%$ patients had a BMI $\geq 30 \mathrm{~kg} / \mathrm{m}^{2}$. In all, $52(62.7 \%)$ patients had optimal surgical debulking, defined as $<1 \mathrm{~cm}$ of residual disease at the conclusion of primary cytoreductive therapy, and $>90 \%$ of patients recurred during the follow-up interval. No association between either age $(P=0.22)$ or race $(P=0.88)$ and frequency of optimal debulking was observed. However, as BMI increased, the frequency of optimal debulking decreased $(P=0.03)$. 
Table 2 Characteristics of study patients

\begin{tabular}{lc}
\hline Characteristic & $\mathrm{n}=83$ \\
\hline Age at diagnosis (years) & \\
Mean (range) & $62.6(34.5-85.9)$ \\
Median & 62.3 \\
Race (\%) & \\
Caucasian & $65(78.3)$ \\
African American & $7(8.4)$ \\
Hispanic & $7(8.4)$ \\
Asian/other & $4(4.9)$ \\
& \\
Body mass index (kg/m $\left.{ }^{2}\right)$ & \\
Mean (range) & $25.9(15.9-50.8)$ \\
Median & 24.3 \\
BMI <25 (\%) & $38(45.8)$ \\
BMI 25 to $<30(\%)$ & $20(24.1)$ \\
BMI $\geq 30(\%)$ & $15(18.1)$ \\
BMI unknown (\%) & $10(12.0)$ \\
& \\
Residual disease after debulking (\%) & \\
Yes & $31(37.3)$ \\
No & $52(62.7)$ \\
Recurrent disease $(\%)$ & \\
Yes & $75(90.4)$ \\
No & $8(9.6)$ \\
Platinum sensitive $(n=52)(\%)^{\mathrm{a}}$ & \\
Yes & $38(73.1)$ \\
No & $14(26.9)$ \\
\hline
\end{tabular}

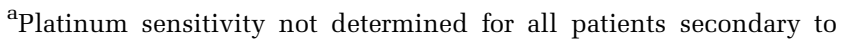
missing/incomplete date information regarding conclusion of adjuvant platinum/taxane chemotherapy.

Table 3 Univariate analysis of overall survival and recurrencefree survival by normalized gene expression $\left(\times 10^{4}\right)$

\begin{tabular}{|c|c|c|c|c|}
\hline \multirow[t]{2}{*}{ Gene } & $\begin{array}{l}\text { Overall } \\
\text { survival }\end{array}$ & \multirow[t]{2}{*}{$\mathrm{P}$-value } & $\begin{array}{l}\text { Recurrence- } \\
\text { free survival }\end{array}$ & \multirow[t]{2}{*}{ P-value } \\
\hline & $H R(95 \% C I)$ & & $H R(95 \%$ CI) & \\
\hline$E R \alpha$ & $0.99(0.94-1.03)$ & 0.54 & $1.02(0.99-1.04)$ & 0.27 \\
\hline EIG121 & $1.21(1.09-1.35)$ & $<0.001$ & $1.13(1.02-1.26)$ & 0.02 \\
\hline$s F R P 1$ & $1.03(0.99-1.06)$ & 0.09 & $1.02(0.98-1.06)$ & 0.32 \\
\hline sFRP4 & $1.00(0.99-1.01)$ & 0.89 & $1.00(0.99-1.01)$ & 0.26 \\
\hline RALDH2 & $1.18(1.03-1.35)$ & 0.02 & $1.06(0.95-1.18)$ & 0.32 \\
\hline$P R$ & $0.99(0.95-1.02)$ & 0.39 & $0.99(0.99-1.01)$ & 0.75 \\
\hline$I G F-1$ & $1.02(0.97-1.07)$ & 0.53 & 1.03 (0.99-1.07) & 0.14 \\
\hline
\end{tabular}

In a univariate analysis, increased expression of EIG121 demonstrated a statistically significant association with worse OS (HR 1.21 (1.09-1.35), $P<0.001$ ) (Table 3). Increased RALDH2 expression was also associated with reduced OS, although to a lesser degree (HR 1.18 (1.03-1.35), $P=0.016)$. There was a trend towards a negative association between sFRP1 expression and OS, but the association was not statistically significant. In a multivariate model that included gene expression and patient clinical characteristics, both sFRP1 (HR 1.04 (1.00-1.07), $P=0.028$ ) and EIG121 (HR 1.20 (CI 1.08-1.35),
$P=0.001)$ remained independently prognostic of worse OS. When RFS was considered, greater EIG121 expression was associated with shorter time to recurrence (HR 1.13 (CI 1.02-1.26), $P=0.021$ ). EIG121 was the only gene in the panel that demonstrated such an effect. When accounting for patient characteristics in a multivariate model, EIG121 remained predictive of RFS (HR 1.14 (CI 1.02-1.27), $P=0.02$ ).

Unsupervised cluster analyses based on gene expression were performed and resulted in a total of 246 unique clusters. All clusters were reviewed, and those that appeared to best segregate patients into two distinct groups were considered for a more detailed analysis. Ultimately, the cluster defined by EIG121 and ER $\alpha$ was selected for further examination because that combination appeared to reasonably segregate tumors into distinct groups of high expressors (cluster 1) and low/intermediate expressors (cluster 2) (Figure 1). The characteristics of the patients in each cluster are described in Table 4. There was no difference between clusters with regard to BMI, age at diagnosis, frequency of optimal debulking, or recurrence. There was a greater proportion of non-White patients in cluster 1 than in cluster 2 ( 41 vs $20 \%, P=0.03$ ).

A univariate analysis of patient characteristics and gene expression clusters in relation to OS is shown in Table 5 . Age at diagnosis $(P=0.56)$, race $(P=0.57)$, and BMI $(P=0.60)$ were not significantly associated with OS. The presence of residual disease at the time of surgical debulking is a classical indicator of poor prognosis in ovarian carcinoma. ${ }^{17}$ In our cohort, we similarly found that the presence of residual disease after surgical debulking significantly reduced OS (HR 2.65 (1.13-6.18), $P=0.02$ ), as did categorization into cluster 1 (HR 3.02 (1.197.65), $P=0.02$ ). In a multivariate analysis, both residual disease (HR 2.53 (1.08-5.95), $P=0.03$ ) and cluster 1 gene expression (HR 2.84 (1.11-7.30), $P=0.03$ ) remained significantly associated with shorter OS. A post hoc statistical evaluation determined that the power to detect an association of this magnitude between cluster 1 gene expression and OS was $99 \%$. The Kaplan-Meier curve depicted in Figure 2 demonstrates significantly different OS between patients in cluster 1 and cluster 2 (log-rank $P=0.014)$.

Table 5 also shows the results of a univariate analysis evaluating gene expression clusters and patient characteristics in relation to RFS. As was seen in OS, age was not associated with RFS $(P=0.87)$. Interestingly, although gene cluster was not predictive of RFS $(P=0.15)$, non-White race (HR 2.01 (1.06-3.84), $P=0.03$ ) residual disease (HR 1.85 (1.07-3.21), $P=0.03$ ), and BMI (HR 1.05 (1.01-1.09), $P=0.02$ ) were all associated with reduced RFS. However, in a multivariate analysis, none of these variables remained statistically significant. As expected, the vast majority $(90 \%)$ of patients in our cohort had recurrent disease after surgical debulking 


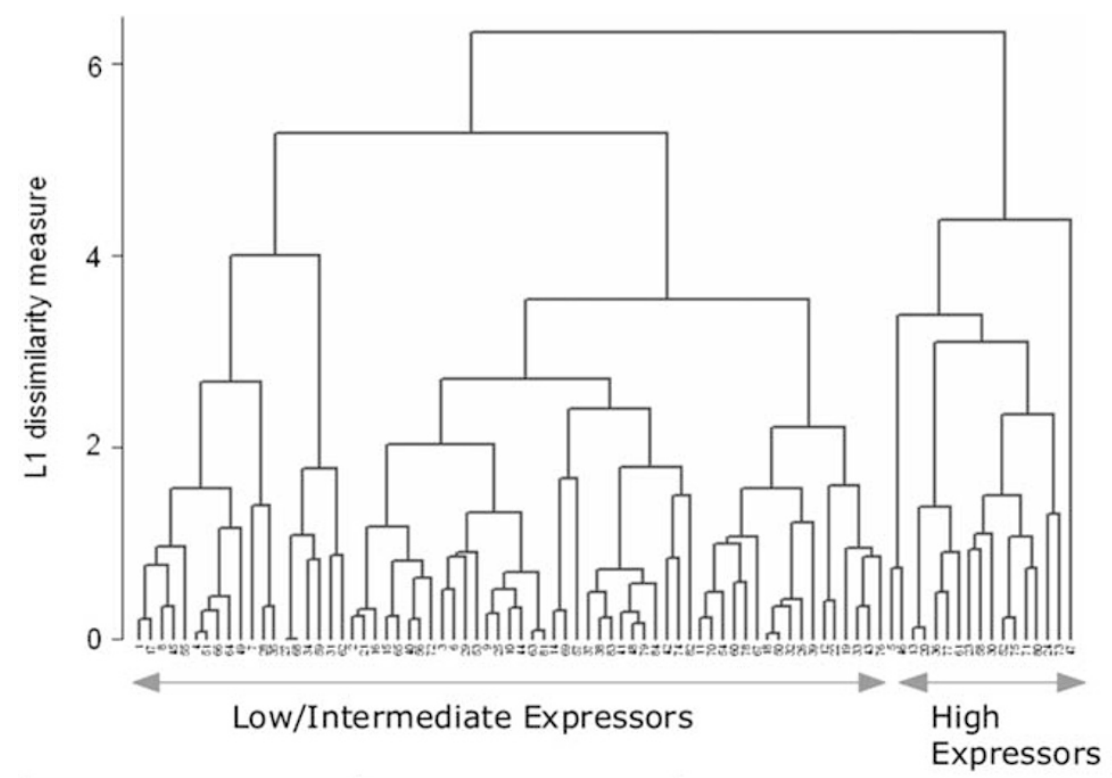

\begin{tabular}{|c|c|c|}
\hline Gene & $\begin{array}{c}\text { High Expression } \\
\text { Cluster } \\
\text { (Cluster } \text { 1) }\end{array}$ & $\begin{array}{c}\text { Low/Intermediate Expression } \\
\text { Cluster } \\
\text { (Cluster 2) }\end{array}$ \\
\hline$E R \alpha$ & 5.18 & 4.24 \\
\hline$E I G 121$ & 3.00 & 0.15 \\
\hline
\end{tabular}

Figure 1 Unsupervised cluster analysis using ER $\alpha$ and EIG121. Cluster 1 has relatively higher expression of these genes, whereas cluster 2 has lower expression. Normalized median gene expression $\left(\times 10^{4}\right)$ is shown in the table below.

Table 4 Patient characteristics by gene cluster

\begin{tabular}{|c|c|c|c|}
\hline Characteristic & $\begin{array}{c}\text { Cluster } 1 \\
\text { (high expressors) } \\
\mathrm{n}=17\end{array}$ & $\begin{array}{c}\text { Cluster } 2 \\
\text { (low/intermediate } \\
\text { expressors) } \mathrm{n}=66\end{array}$ & $\mathrm{P}$-value \\
\hline \multicolumn{4}{|c|}{ Age at diagnosis (years) } \\
\hline Median & 60.1 & 63.3 & 0.42 \\
\hline \multicolumn{4}{|c|}{ Body mass index $\left(\mathrm{kg} / \mathrm{m}^{2}\right)$} \\
\hline Mean & 25.4 & 28.3 & 0.12 \\
\hline Range & $15.9-50.8$ & $17.9-47.3$ & \\
\hline \multicolumn{4}{|l|}{ Race (\%) } \\
\hline White & $10(58.8)$ & $55(83.3)$ & 0.03 \\
\hline Non-White & $7(41.2)$ & $11(16.7)$ & \\
\hline \multicolumn{4}{|c|}{ Optimal debulking (\%) } \\
\hline Yes & $9(52.9)$ & $44(67.7)$ & 0.14 \\
\hline No & $8(47.1)$ & $22(33.3)$ & \\
\hline \multicolumn{4}{|c|}{ Recurrent disease (\%) } \\
\hline Yes & $14(82.4)$ & $61(92.4)$ & 0.75 \\
\hline No & $3(17.6)$ & $5(4.6)$ & \\
\hline \multicolumn{4}{|c|}{ Platinum sensitive $(n=52)(\%)^{\mathrm{a}}$} \\
\hline Yes & $7(18.4)$ & $31(81.6)$ & 1.00 \\
\hline No & $3(21.4)$ & $11(78.6)$ & \\
\hline
\end{tabular}

${ }^{\text {a }}$ Platinum sensitivity not determined for all patients secondary to missing/incomplete date information regarding conclusion of adjuvant platinum/taxane chemotherapy.

and first-line chemotherapy. It is possible that the wide variety of treatment approaches used for recurrent disease is obscuring any effects of gene cluster and other variables on RFS.
ER immunohistochemistry is currently the accepted standard method of determining a tumor's potential sensitivity to ER antagonists or antiestrogen agents. Overall, $88 \%$ of all tumors in this study demonstrated strong $(2+$ or $3+)$ positive ER staining in $>10 \%$ of tumor cell nuclei. The mean percentage of ER-positive cells per tumor was not significantly different between cluster 1 and cluster 2 (69 vs $59 \%, P=0.29$ ). When ER expression was categorized as either negative $(\leq 10 \%)$ or positive $(>10 \%)$, the proportion of samples staining positive was not significantly different between gene clusters $(P=0.14)$. Finally, ER expression by immunohistochemistry did not correlate with gene cluster assignment $(R=0.29, P=0.1)$.

The results of ER immunohistochemistry were assessed to determine whether percentage of ER-positive tumor cells correlated with survival. When percentage of ER-positive tumor cells is analyzed as a continuous variable to predict outcomes, there is no association between percentage of ER-positive cells and either OS (HR 1.00, $P=0.89$ ) or RFS (HR $0.99, P=0.60$ ). The mean percentage of ER-positive tumor cells was quite high overall (62 $\pm 5.0 \%$; mean \pm s.e.). Using $30 \%$ ER-positive tumor cells as a cutoff (because this was the lowest value to generate adequate groups for comparison), univariate analysis demonstrated no significant difference in either OS (HR $1.20(0.33-4.30), P=0.78)$ or RFS (HR $0.70(0.27-1.80), P=0.46)$. Using 10 or $20 \%$ ERpositive cells as cutoffs similarly yielded statistically nonsignificant findings (data not shown); 
Table 5 Univariate analysis of overall survival and recurrence-free survival by patient characteristics

\begin{tabular}{|c|c|c|c|c|}
\hline \multirow[t]{2}{*}{ Variable } & Overall survival & \multirow[t]{2}{*}{ P-value } & Recurrence-free survival & \multirow[t]{2}{*}{ P-value } \\
\hline & HR (with 95\% CI) & & HR (with 95\% CI) & \\
\hline Age at diagnosis (months) & $1.00(0.99-1.01)$ & 0.56 & $1.00(0.99-1.00)$ & 0.87 \\
\hline Race (White vs non-White) & $1.33(0.49-3.62)$ & 0.57 & $2.01(1.06-3.81)$ & 0.03 \\
\hline Residual disease after debulking surgery & $2.65(1.13-6.18)$ & 0.02 & $1.85(1.07-3.21)$ & 0.03 \\
\hline Post-operative BMI & $1.02(0.95-1.09)$ & 0.60 & $1.05(1.01-1.09)$ & 0.02 \\
\hline Cluster (1 vs 2) & $3.02(1.19-7.65)$ & 0.02 & $1.65(0.84-3.22)$ & 0.15 \\
\hline
\end{tabular}

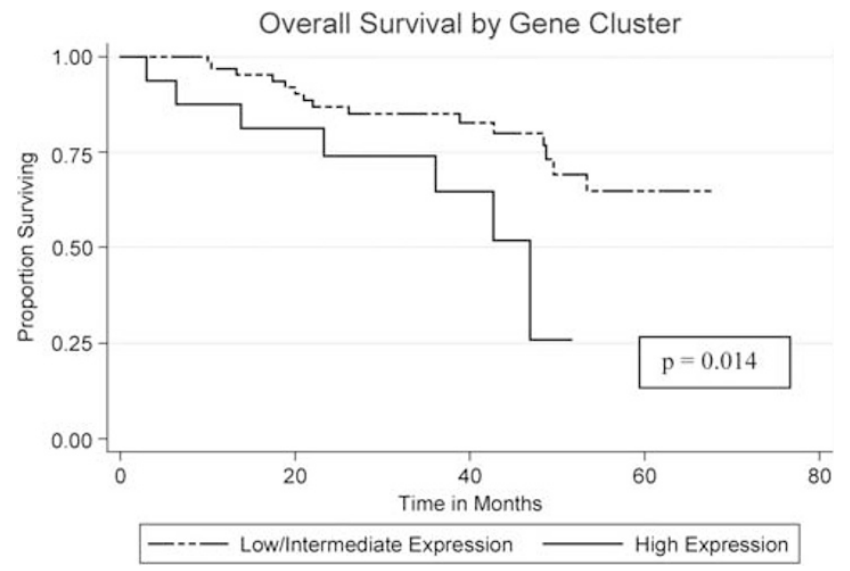

Figure 2 The Kaplan-Meier curve of overall survival by gene cluster. Patients in cluster 1 (high gene expression) had a significant overall survival disadvantage.

however, the number of subjects in the ER-low group for each of these cutoffs was extremely low. ER immunohistochemical expression was not significantly associated with patient age, BMI, race, surgical debulking status, recurrence, or platinum sensitivity (data not shown).

\section{Discussion}

Estrogen is a potent steroid hormone responsible for normal physiological functions in women, including ovulation and the maintenance of bone mineral density. In the setting of aberrations to normal physiology, most notably in the setting of a cancer diagnosis, estrogen takes on a different role. For hormone-sensitive malignancies, estrogen can stimulate cancer cell proliferation. However, the presence of estrogen sensitivity may also be a positive prognostic marker. In both endometrial and breast cancers, higher expression of estrogeninduced genes is associated with tumors that tend to be low grade and less biologically aggressive. ${ }^{10,11}$ Greater estrogen sensitivity and increased expression of genes induced by estrogen are also predictive of improved survival. ${ }^{10,11,18}$ Importantly, the identification of tumor estrogen sensitivity allows physicians to consider using hormone antagonistic treatments, which are less toxic than traditional chemotherapy and less significantly impact patient quality of life.

The fact that ER immunohistochemistry did not correlate well with the gene-based clustering approach of high-grade serous ovarian carcinoma is important. From previous clinical trials of tamoxifen and aromatase inhibitors in women with ovarian cancer, we can estimate a response rate of $13-26 \% .^{6,8,9}$ However, as shown in this study, $88 \%$ of high-grade serous ovarian carcinomas have sufficient immunohistochemical expression of ER to be considered 'positive' and thus eligible for tamoxifen, letrozole, or other similar inhibitors of estrogen action. Thus, ER immunohistochemistry is significantly overestimating the subgroup of ovarian cancer patients who may benefit from such hormonal approaches. In contrast, the approach of using transcript quantification of ER $\alpha$ and EIG121 of this same set of tumors demonstrated that only $20 \%$ of these patients had higher expression of $E R \alpha$ and EIG121; this figure more closely approximates the $13-26 \%$ response rate of estrogen-directed therapies for ovarian cancer. Therefore, this provides good preliminary evidence that quantification of transcripts from a gene panel may be a more accurate clinical method of identifying women who would benefit most from such estrogen-directed therapeutic approaches. However, a prospective clinical trial will be necessary to definitively prove this point.

Abundant data exist demonstrating the poor prognostic capabilities of hormone receptor immunohistochemistry for ovarian cancer. ${ }^{19-25}$ For example, Slotman et $a l^{21}$ observed that there was no correlation between ER expression and overall patient survival in a group of women with ovarian carcinoma, whereas other authors have argued that combined ER/PR expression can distinguish between groups of patients with markedly different survivals. ${ }^{20}$ Even when used to gauge potential response to hormonal antagonistic therapies, receptor positivity correlates poorly with treatment outcomes. Rose et $a l^{23}$ examined 123 ovarian carcinomas for PR and ER expression between 1981 and 1989. In a population of mixed histologies, ER and PR were not significantly associated with survival, chemotherapy response, or second-look findings in a multivariate analysis. An alternative 
approach, quantification of panels of transcripts, is already widely used in the management of breast cancer. Oncotype DX, a multigene classifier that analyzes RNA expression from paraffin-embedded tumor tissue, is currently recommended by the American Society of Clinical Oncology in earlystage breast cancer as a prognostic tool to both determine the risk for disease recurrence and predict the response to treatment. ${ }^{26,27} \mathrm{~A}$ second gene classifier for breast cancer, MammaPrint, is currently under investigation, but it has shown similar prognostic capability. ${ }^{27}$

In the current study, it was initially hypothesized that improved survival would be seen in women whose ovarian tumors expressed high levels of $E R \alpha$ and estrogen-induced genes. However, this was not observed. High expression of $E R \alpha$ and estrogeninduced genes was not only associated with worse OS but it was also a negative prognostic factor independent of other patient-dependent covariates, such as age, race, and BMI. Such a finding suggests that the molecular mechanisms underlying ovarian tumorigenesis may in fact be quite different in highgrade serous ovarian cancer compared with hormone-sensitive tumors at other disease sites, or perhaps even different ovarian histologies. It is known that exposure to unopposed estrogen is associated with an increased risk of developing ovarian carcinoma. ${ }^{28}$ However, more detailed epidemiological studies have shown that a specific type of ovarian carcinoma, the less common histotype endometrioid adenocarcinoma, is most closely linked to estrogen exposure, whereas high-grade serous carcinoma is not linked to estrogen exposure. ${ }^{29}$ Therefore, the findings reported for our current study may be specific for ovarian high-grade serous carcinoma and not applicable to endometrioid-type ovarian tumors.

There is some experimental data that suggest that estrogen and/or genes induced by estrogen may actually promote adverse biological properties of ovarian cancer cells. Murdoch and Van Kirk ${ }^{30}$ showed that ovarian cancer cells treated with estrogen had a significantly decreased ability to undergo apoptosis. In addition, after causing cellular stress by treatment with cisplatin, ovarian cancer cells exposed to estrogen had significantly increased DNA repair activity. ${ }^{30}$ The authors suggested that estrogen antagonized the apoptotic pathway triggered by chemotherapy-induced DNA damage. Although the pathways by which estrogen achieved these changes were not specifically elucidated, these findings are significant because platinum drugs are one of two primary agents used for the treatment of ovarian carcinoma, including highgrade serous carcinoma.

Estrogen's role in stimulating ovarian cancer progression may also lie in the activation of nongenomic signaling mechanisms. Park et $a l^{31}$ found that when estrogen-sensitive ovarian cancer cell lines are exposed to estrogen, cells undergo an epithelial-to-mesenchymal transition in which they lose expression of E-cadherin, become more spindle shaped, and develop pseudopodia. ${ }^{31}$ Estrogen-associated cross-talk with the epidermal growth factor receptor (EGFR) pathway may further promote ovarian tumor progression. In ovarian cancer, EGFR expression is a negative prognostic indicator of survival, and overexpression is associated with poor responses to chemotherapy in both in vitro and clinical studies. ${ }^{32,33}$ Research on other estrogen-sensitive tissues suggests potential mechanisms to explain this phenomenon. McBean et $a l^{34}$ reported that estrogen treatment increased endometrial tissue expression of EGFR mRNA and EGFR protein by IHC. Filardo ${ }^{35}$ later described a signaling cascade in breast cancer cells initiated by estrogen's interaction with a membrane-bound G protein-coupled receptor that subsequently activated EGFR and downstream Mek. ${ }^{35}$

EIG121 was significantly associated with poorer OS in our cohort and has been investigated as a prognostic marker in other hormone-sensitive tumors. Interestingly, in both breast and endometrial cancers, increased expression of EIG121 predicts improved survival. ${ }^{10,11}$ Recent mechanistic studies suggest a function of EIG121, which may help to support our current findings that its increased expression in ovarian cancer is associated with poor outcome. $^{36}$ In particular, the EIG121 protein is localized to the late endosome-lysosome compartments and regulates autophagy, a cellular prosurvival mechanism activated when a cell is stressed by a lack of nutrients or chemotherapy. ${ }^{36}$ It was shown that EIG121 conferred protection against the induction of apoptosis on cells exposed to serum starvation and treatment with taxane chemotherapy. ${ }^{36}$

When considering these possible mechanisms to explain our findings of decreased survival associated with increased expression of ER $\alpha$ and EIG121, it is important to also keep in mind that first-line therapy of ovarian high-grade serous carcinoma is very different from that of ER-positive breast carcinoma or endometrioid-type endometrial carcinoma. Many low-grade endometrioid-type endometrial carcinomas are treated with surgery alone; chemotherapy is reserved for recurrences or when there are metastases outside the uterus. Many ER-positive breast carcinomas are well differentiated and respond well to tamoxifen or other pharmacological methods to antagonize estrogen's action. It is possible that potential hormonal responsiveness in ovarian high-grade serous carcinoma is masked by the front-line use of cytotoxic chemotherapy.

Identifying biomarkers is important to adequately counsel individual patients regarding their prognoses, but biomarkers may also provide insights into potential therapeutic interventions. Given the finding that high expression of ER $\alpha$ and estrogeninduced genes in high-grade serous ovarian tumors is associated with a poorer prognosis, and that the downstream products of estrogen stimulation may 
enhance cancer cell survival, it is reasonable to consider the addition of an estrogen antagonist to current treatment regimens. At present, the standard of care for ovarian carcinoma is therapy with platinum and taxane agents, both of which are cytotoxic and function independently of hormonemediated pathways..$^{37,38}$ In selected patients with high ER $\alpha$ and EIG121 expression, supplementing this regimen with an estrogen-antagonizing drug may prevent ovarian cancer cells from activating self-preservation pathways, thus making them more susceptible to the lethal effects of chemotherapeutic agents.

\section{Acknowledgements}

This study was supported by NIH T32 Training Grant (MPS), NIH 1P50CA098258-01 SPORE in Uterine Cancer (RRB), and NIH P50CA83639 SPORE in Ovarian Cancer (RRB). This original research was presented in part at the 99th United States and Canadian Academy of Pathology Annual Meeting, 20-26 March 2010, Washington, DC, and at the 41st Society of Gynecologic Oncologists Annual Meeting on Women's Cancer, 13-17 March 2010, San Francisco, CA. The authors would like to thank Sally W Vernon, PhD, and Thomas H Stock, PhD, $\mathrm{MPH}$, for their continued support during the completion of this manuscript.

\section{Disclosure/conflict of interest}

The authors declare no conflict of interest.

\section{References}

1 Jemal A, Siegel R, Ward E, et al. Cancer statistics, 2009. CA Cancer J Clin 2009;59:225-245.

2 Aghajanian C, Blessing J, Darcy K, et al. A phase II evaluation of bortezomib in the treatment of recurrent platinum-sensitive ovarian or primary peritoneal cancer: a Gynecologic Oncology Group study. Gynecol Oncol 2009;115:215-220.

3 Schorge J, Schaffer J, Halvorson L, et al (eds) Williams Gynecology. McGraw Hill: New York, 2008.

4 Hunt K, Robb G, Strom E, et al (eds). Breast Cancer, 2nd edn., Springer: New York, 2008.

5 Rao BR, Slotman BJ. Endocrine role in ovarian cancer. Endocr Relat Cancer 1996;3:309-326.

6 Perez-Gracia J, Carrasco E. Tamoxifen therapy for ovarian cancer in the adjuvant and advanced setting: systematic review of the literature and implications for future research. Gynecol Oncol 2002;84:201-209.

7 Schwartz P, Chambers J, Kohort E, et al. Tamoxifen in combination with cytotoxic chemotherapy in advanced epithelial ovarian cancer. A prospective randomized trial. Cancer 1989;63:1074-1078.

8 Ramirez P, Schmeler K, Milam M, et al. Efficacy of letrozole in the treatment of recurrent platinum- and taxane-resistant high-grade cancer of the ovary or peritoneum. Gynecol Oncol 2008;110:56-59.

9 Smyth J, Gourley C, Walker G, et al. Antiestrogen therapy is active in selected ovarian cancer cases: the use of letrozole in estrogen-receptor positive patients. Clin Cancer Res 2007;13:3617-3622.

10 Oh D, Troester M, Usary J, et al. Estrogen-regulated genes predict survival in hormone receptor-positive breast cancers. J Clin Oncol 2006;24:1656-1664.

11 Westin S, Broaddus R, Deng L, et al. Molecular clustering of endometrial carcinoma based on estrogen-induced gene expression. Cancer Biol Ther 2009; 8:2126-2135.

12 Johns Hopkins Pathology. Ovarian Cancer November 2001 [cited 18 May 2010]; Available from:http:// ovariancancer.jhmi.edu/treatment.cfm.

13 Deng L, Broaddus R, McCampbell A, et al. Identification of a novel estrogen-regulated gene, EIG121, induced by hormone replacement therapy and differentially expressed in type I and type II endometrial cancer. Clin Cancer Res 2005;11:8258-8264.

14 Deng L, Shipley G, Loose-Mitchell D, et al. Coordinate regulation of the production and signaling of retinoic acid by estrogen in the human endometrium. J Clin Endocrinol Metab 2003;88:2157-2163.

15 O'Toole S, Dunn E, Sheppard B, et al. Oestrogen regulated gene expression in normal and malignant endometrial tissue. Maturitas 2005;51:187-198.

16 Rutanen E. Insulin-like growth factors in endometrial function. Gynecol Endocrinol 1998;12:399-406.

17 Winter W, Maxell G, Tian C, et al. Tumor residual after surgical cytoreduction in prediction of clinical outcome in stage IV epithelial ovarian cancer: a Gynecologic Oncology Group Study. J Clin Oncol 2008; 26:83-89.

$18 \mathrm{Yu} \mathrm{J,} \mathrm{Yu} \mathrm{J,} \mathrm{Cordero} \mathrm{K,} \mathrm{et} \mathrm{al.} \mathrm{A} \mathrm{transcriptional}$ fingerprint of estrogen in human breast cancer predicts patient survival. Neoplasia 2008;10:79-88.

19 Geisler J, Wiemann M, Miller G, et al. Estrogen and progesterone receptor status as prognostic indicators in patients with optimally cytoreduced stage IIIc serous cystadenocarcinoma of the ovary. Gynecol Oncol 1996;60:424-427.

20 Arias-Pulido H, Smith H, Joste N, et al. Estrogen and progesterone receptor status and outcome in epithelial ovarian cancers and low malignant potential tumors. Gynecol Oncol 2009;114:480-485.

21 Slotman B, Kuhnel R, Rao B, et al. Importance of steroid receptors and aromatase activity in the prognosis of ovarian cancer: high tumor progesterone levels correlate with longer survival. Gynecol Oncol 1989; 33:76-81.

22 Munstedt K, Steen J, Knauf A, et al. Steroid hormone receptors and long term survival in invasive ovarian cancer. Cancer 2000;89:1783-1791.

23 Rose P, Reale F, Longcope C, et al. Prognostic significance of estrogen and progesterone receptors in epithelial ovarian cancer. Obstet Gynecol 1990;76: 258-263.

24 Tangjitgamol S, Manusirivithaya S, Khunnarong J, et al. Expressions of estrogen and progesterone receptors in epithelial ovarian carcinoma: a clinicopathologic study. Int J Gynecol Cancer 2009;19:620-627.

25 Hogdall E, Christensen L, Hogdall C, et al. Prognostic value of estrogen receptor and progesterone receptor tumor expression in Danish ovarian cancer patients: 
from the 'MALOVA' Ovarian Cancer Study. Oncol Rep 2007;18:1051-1059.

26 Ross J. Multigene classifiers, prognostic factors, and predictors of breast cancer clinical outcome. Adv Anat Pathol 2009;16:204-215.

27 Dobbe E, Gurney K, Kiekow S, et al. Gene-expression assays: new tools to individualize treatment of earlystage breast cancer. Am J Health Syst Pharm 2008;65: 23-28.

28 Danforth K, Tworoger S, Hecht J, et al. A prospective study of postmenopausal hormone use and ovarian cancer risk. Br J Cancer 2007;96:151-156.

29 Purdie D, Bain C, Siskind V, et al. Hormone replacement therapy and risk of epithelial ovarian cancer. Br J Cancer 1999;81:559-563.

30 Murdoch W, Van Kirk E. Oestradiol inhibits spontaneous and cisplatin-induced apoptosis in epithelial ovarian cancer cells: relationship to DNA repair capacity. Apoptosis 1997;2:478-484.

31 Park S, Cheung L, Wong A, et al. Estrogen regulates snail and slug in the down-regulation of E-cadherin and induces metastatic potential of ovarian cancer cells through estrogen receptor alpha. Mol Endocrinol 2008;22:2085-2098.

32 Fischer-Colbrie J, Witt A, Heinzl H, et al. EGFR and steroid receptors in ovarian carcinoma: comparison with prognostic parameters and outcome of patients. Anticancer Res 1997;17:613-619.

33 Bull Phelps S, Schorge J, Peyton M, et al. Implications of EGFR inhibition in ovarian cancer cell proliferation. Gynecol Oncol 2008;109:411-417.

34 McBean J, Brumsted J, Stirewalt W. In vivo estrogen regulation of epidermal growth factor receptor in human endometrium. J Clin Endocrinol Metab 1997; 82:1467-1471.

35 Filardo E. Epidermal growth factor receptor (EGFR) transactivation by estrogen via the G-protein-coupled receptor, GPR30: a novel signaling pathway with potential significance for breast cancer. J Steroid Biochem Mol Biol 2002;80:231-238.

36 Deng L, Feng J, Broaddus R. The novel estrogeninduced gene EIG121 regulates autophagy and promotes cell survival under stress. Cell Death Dis 2010;1:e32.

37 McGuire W, Hoskins W, Brady M, et al. Cyclophosphamide and cisplatin compared with paclitaxel and cisplatin in patients with stage III and stage IV ovarian cancer. N Engl J Med 1996;334:1-6.

38 Piccart M, Bertelsen K, James K, et al. Randomized intergroup trial of cisplatin-paclitaxel versus cisplatincyclophosphamide in women with advanced epithelial ovarian cancer: three-year results. J Natl Cancer Inst 2000;92:699-708. 